\title{
Current Targets of Immune Suppression in Tumor Microenvironment
}

\author{
Kwei-Yan Liu ${ }^{1}$, Li-Ting Wang ${ }^{1}$ and Shih-Hsien Hsu ${ }^{1,2 *}$ \\ ${ }^{1}$ Graduate Institute of Medicine, Kaohsiung Medical University, Taiwan \\ ${ }^{2}$ Department of Medical Research, Kaohsiung Medical University Hospital, Taiwan
}

*Corresponding author: Shih-Hsien Hsu, Graduate Institute of Medicine, Kaohsiung Medical University, 807 Kaohsiung, Taiwan

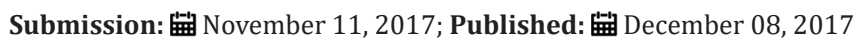

\begin{abstract}
Opinion
In the past years, scientists have discovered tumor microenvironment (TME) as a comfort zone of cancer cells. Efforts are being globally made to disrupt this comfort zone of cancer cells. However, a despiteseveral effort, the complete blockage of tumor progression has not been achieved because of unclear cellular and molecular mechanisms of TME crosstalk. Cancer immunotherapy is one of the promising therapies that have the ability to disrupt TME by restoring the immune attack on malignant cells [1]. However, positive outcomes of immunotherapy have been reported only in few patients, suggesting a need for the unmasking of regulatory mechanisms.
\end{abstract}

In TME, tumor cells express programmed cell-death 1ligand 1(PD-L1) and cluster of differentiation 86 (CD86) as ligands binding to immune-checkpoint receptors. These ligands bind to programmed cell-death protein 1 (PD-1) and cytotoxic T-lymphocyte-associated protein 4 (CTLA-4) of CD8+ T-cell to limit host immune responses [2]. Hence, targeting CTLA-4 and CD86 or PD-1 and PD-L1has emerged as a new strategy of cancer immune therapies in recent years and has demonstrated clinical efficacy. In our previous studies, we demonstrated that liver tumors result in immune suppression by expressing PD-L1 and CD86.Further, these expressions were induced by the transcriptional factor ISX, encoded by intestine-specific homeoboxgene [3]. The knockdown of ISX in liver tumors reverses the immune activity of CD8+ T-cells and promotes the death of liver tumor cells. In contrast, the blockage of PD-L1 only resulted ina partial inhibition of tumor growth and metastasis. These results confirmed that ectopic oncogene expression (e.g ISX) activates multiple immune suppression pathways in addition to immune-checkpoint suppression.

Our earlier reports have confirmed that ISX also contributes to the kynurenine (KYN)-aryl hydrocarbon receptor (AHR) axis of tumor immune suppression.AHR is a ligand-activated transcription factor that plays divergent roles in tumor and immune cells. It acts as a cellular chemical sensor activated by numerous environmental pollutants, including polycyclic aromatic hydrocarbons and diverse endogenous metabolites [4]. In TME, KYN accumulation, endogenous metabolites of tryptophan catabolism, as a ligand activates AHR, which translocates to the nucleus and results in the transcription of target genes [5]. AHR activation in tumor cells resulted in an increase in the survival and motility of tumor cells, whereas AHR activation in immune cells caused the arrest and tolerance of immune cells. In liver tumor, weid entified the ISX-KYNAHR axis as a self-perpetuating loop conferring tumorigenic effects and exerting its immune suppressive effects. ISX is a KYN-AHR target gene, the expression of which enhances KYN accumulation in TME by the direct transcriptional regulation of tryptophan catabolic enzymes in doleamine 2,3-dioxygenases 1(ID01) and tryptophan 2,3-dioxygenase (TDO2). AHR activation by KYN or ISX activation by integrating IL- 6 signaling could amplify this feedback loop to build up immune-checkpoint-and AHR-dependent immune suppressive effect in TME.

Currently, in cancer immune therapy, the mere targeting of immune-checkpoint receptor is insufficient to entirely disrupt TME. Our studies identified that targeting the ISX-KYN-AHR axis impairs TME and is a potential target for rescuing both immunecheckpoint- and AHR-dependent immune suppressive effects. Therefore, we propose that targeting both immune-checkpointand AHR-dependent immune suppressive effects will result in an efficient immunotherapeutic approach against cancer in future.

\section{References}

1. Beatty GL, Gladney WL (2015) Immune escape mechanisms as a guide for cancer immunotherapy. Clin Cancer Res 21(4): 687-692.

2. Smyth MJ, Ngiow SF, Ribas A, Teng MW (2016) Combination cancer immunotherapies tailored to the tumour microenvironment. Nat Rev Clin Oncol 13(3): 143-158.

3. Wang LT, Chiou SS, Chai CY, Hsi E, Yokoyama KK, et al. (2017) IntestineSpecific Homeobox Gene ISX Integrates IL6 Signaling, Tryptophan Catabolism, and Immune Suppression. Cancer Res 77(15): 4065-4077.

4. Murray IA, Patterson AD, Perdew GH (2014) Aryl hydrocarbon receptor ligands in cancer: friend and foe. Nat Rev Cancer 14(12): 801-814.

5. Platten M, Wick W, Van den Eynde BJ (2012) Tryptophan catabolism in cancer: beyond IDO and tryptophan depletion. Cancer Res 72(21): 54355440 . 